\title{
Therapeutic Options for the Management of the Cardiorenal Syndrome
}

\author{
Katerina Koniari, Marinos Nikolaou, Ioannis Paraskevaidis, and John Parissis
}

Heart Failure Unit, 2nd Cardiology Department, Attikon University Hospital, University of Athens, Athens, Greece

Correspondence should be addressed to Katerina Koniari, ekoniari@med.uoa.gr

Received 17 August 2010; Accepted 11 October 2010

Academic Editor: Mitchell H. Rosner

Copyright (๑) 2011 Katerina Koniari et al. This is an open access article distributed under the Creative Commons Attribution License, which permits unrestricted use, distribution, and reproduction in any medium, provided the original work is properly cited.

\begin{abstract}
Patients with heart failure often present with impaired renal function, which is a predictor of poor outcome. The cardiorenal syndrome is the worsening of renal function, which is accelerated by worsening of heart failure or acute decompensated heart failure. Although it is a frequent clinical entity due to the improved survival of heart failure patients, still its pathophysiology is not well understood, and thus its therapeutic approach remains controversial and sometimes ineffective. Established therapeutic strategies, such as diuretics and inotropes, are often associated with resistance and limited clinical success. That leads to an increasing concern about novel options, such as the use of vasopressin antagonists, adenosine A1 receptor antagonists, and renalprotective dopamine. Initial clinical trials have shown quite encouraging results in some heart failure subpopulations but have failed to demonstrate a clear beneficial role of these agents. On the other hand, ultrafiltration appears to be a more promising therapeutic procedure that will improve volume regulation, while preserving renal and cardiac function. Further clinical studies are required in order to determine their net effect on renal function and potential cardiovascular outcomes. Until then, management of the cardiorenal syndrome remains quite empirical.
\end{abstract}

\section{Introduction}

Renal dysfunction is one of the most important comorbidities in heart failure. Decreased estimated glomerular filtration rate (GFR) seems to be a potent predictor of cardiovascular complications and mortality [1]. In addition, worsening heart failure or acute decompensated heart failure (ADHF) can accelerate worsening of renal function, that is what we call cardiorenal syndrome (CRS). The most common underlying risk factors that account for renal dysfunction in the setting of heart failure or cardiac dysfunction include hypertension, diabetes mellitus, severe atherosclerotic disease, elderly age, and a prior history of renal insufficiency or heart failure [2].

As patients with heart failure are surviving much longer and dying less frequently from primary arrhythmia, we suppose that the CRS will become more common in the near future. However, there is no a single definition that appropriately describes this entity. It is well accepted that cardiovascular morbidity and mortality and diminished renal function are closely correlated. This relationship exists regardless of whether the initial event is a parenchymal disease of the kidney or a cardiac disease. In SOLVD (Studies of Left Ventricular Dysfunction) trial, patients with a GFR less than $60 \mathrm{ml} /$ minute $/ 1.73 \mathrm{~m}^{2}$ had a $40 \%$ higher risk of death $[3,4]$. In addition, in the ADHERE (Acute Decompensated Heart Failure National Registry) population, mortality risk for the hospitalized patients could be estimated using three variables: systolic blood pressure, blood urea nitrogen (BUN), and serum creatinine levels. Two of the above three most important predictors of in-hospital survival are related to kidney function [5]. Similarly, Gottlieb et al. showed that in hospitalized patients, worsening renal function predicts a prolonged hospitalization or an increased risk of death [6].

The current proposed definition divides CRS into five subtypes: type I, acute CRS (20-25\%), which reflects an abrupt worsening of cardiac function (e.g., acute cardiogenic shock or acutely decompensated congestive heart failure) leading to acute kidney injury; type II, chronic CRS (30$45 \%)$, in which chronic abnormalities in cardiac function 
(e.g., chronic congestive heart failure) cause progressive and potentially permanent chronic kidney disease; type III, acute renocardiac syndrome (30-35\%), which reflects an abrupt worsening of renal function (e.g., acute kidney ischaemia or glomerulonephritis) leading to acute cardiac disorder (e.g., heart failure, arrhythmia, or ischemia); type IV, chronic renocardiac syndrome (45-50\%), in which chronic kidney disease (e.g., chronic glomerular or interstitial disease) contributes to decreased cardiac function, cardiac hypertrophy, and/or increased risk of adverse cardiovascular events; and type V, secondary CRS, meaning systemic diseases such as diabetes mellitus, sepsis, and amyloidosis that deteriorate simultaneously cardiac and renal function $[7,8]$.

\section{Pathophysiology of the CRS}

Heart and kidney performance are closely interrelated physiologically and pathophysiologically, both in health and in disease. Although there is a growing recognition of the frequent presentation of the CRS, its underlying pathophysiology is not yet well understood, and no consensus regarding its appropriate management has been achieved.

A decreased cardiac output in CHF resulting in reduced renal perfusion could be an easy explanation for the worsening renal function. But worsening renal function has also been demonstrated among patients with ADHF with preserved left ventricular ejection fraction. This deterioration in renal performance, despite a presumed preservation of blood flow to the kidneys, has led to the search of other pathophysiological mechanisms [9]. Although the pathophysiology varies according to the specific clinical circumstances, the general processes include neurohormonal factors and hemodynamic factors, such as intrarenal hemodynamics and transrenal perfusion pressure.

Transrenal perfusion pressure is estimated as mean arterial pressure minus central venous pressure. For the patient with heart failure and volume overload, the combination of high pulmonary artery or central venous pressure with low systemic pressure may cause a severe compromise of the net renal perfusion pressure. Therefore, when there is an opportunity to decrease central venous pressure through vasodilatation, improved oxygenation, or volume reduction, this can lead to significant improvements in renal blood flow and urine output [2].

Moreover, a very important contributor is the neurohormonal activation, which is mediated by activation of arterial baroreceptors and intrarenal sensors (Figure 1). That leads to exaggerated abnormalities in the activation of the reninangiotensin-aldosterone system (RAAS), activation of the sympathetic nervous system (SNS), and also activation of the arginine-vasopressin system. The latter is an intrinsic selfdefense system that maintains blood pressure and intravascular volume within normal range. Besides vasoconstriction and sodium retention that lead to increased preload and afterload, one of the most deleterious actions of the RAAS in CRS is the activation of NADPH-oxidase by angiotensin II. This results in the formation of reactive oxygen species (ROS). In CRS, there is no balance between NO and ROS because of the increased production of the latter. A major initiator of an inflammatory response is oxidative stress through the production and activation of proinflammatory cytokines, especially interleukin-1, interleukin-6, C-reactive protein, and tumor necrosis factor-a. It is well known that these cytokines play a crucial role in the pathophysiology of atherosclerosis. Moreover, they have negative inotropic effects, assist in cardiac remodeling, and cause thrombotic complications. Therefore, a vicious cycle sets in, promoting structural and functional damage to the heart and to the kidneys $[10,11]$.

The production of endothelin has also some adverse effects because it causes vasoconstriction and enhances hypertrophy of cardiac myocytes. Moreover, it stimulates noradrenaline, angiotensin II, and aldosterone [12].

Arginine vasopressin (AVP) has also detrimental effects on CRS progression by fluid retention and enhancement of angiotensin II and noradrenaline actions. In addition it simulates myocardial hypertrophy [13].

Adenosine and the related tubuloglomerular feedback is a recently identified contributing factor. Adenosine is locally released in the kidney under stress. It binds to receptors on the afferent arterioles and promotes vasoconstriction, thereby reducing renal blood flow. Activation of the receptor also enhances sodium reabsorption in the tubules, leading to further water and sodium retention. Acute delivery of sodium to the distal tubules due to diuretic therapy in ADHF will in turn stimulate further adenosine release and further reduction in the GFR. This pathway might be very attractive as a contemporary therapeutic target in CRS [14].

In heart failure, the SNS is initially activated by the baroflex to provide inotropic support and preserve cardiac output. However, excessive sympathetic activity can enhance cardiomyocyte apoptosis and focal myocardial necrosis, while the direct actions of catecholamines can lead to hypertrophy [11]. Finally, the aggressive use of diuretic agents may cause further neurohormonal activation and aggravate systemic and renal vasoconstriction, leading to additional impairment in renal performance. The consequent decline in blood flow and filtration contribute actively to the clinical entity of diuretic resistance [2].

\section{Treatment of Patients with CRS}

The heterogeneous and complex pathophysiology of CRS makes patient management a clinical challenge for the physicians. To date there is not a single success-guaranteed treatment for CRS because of two main reasons. The first one is that each patient has his own unique medical history, risk profile, and combination of comorbidities. The second one is that we have no evidence from clinical heart failure trials on which we can base our therapy for patients with significant renal dysfunction since most studies predominantly recruited populations with relatively preserved renal function [15]. Another serious point in the therapeutic approach of patients with CRS is the development of resistance to many standard therapies, such as diuretics and inotropes, which leads to an increasing concern about novel strategies (e.g., use of AVP antagonists, adenosine A1 


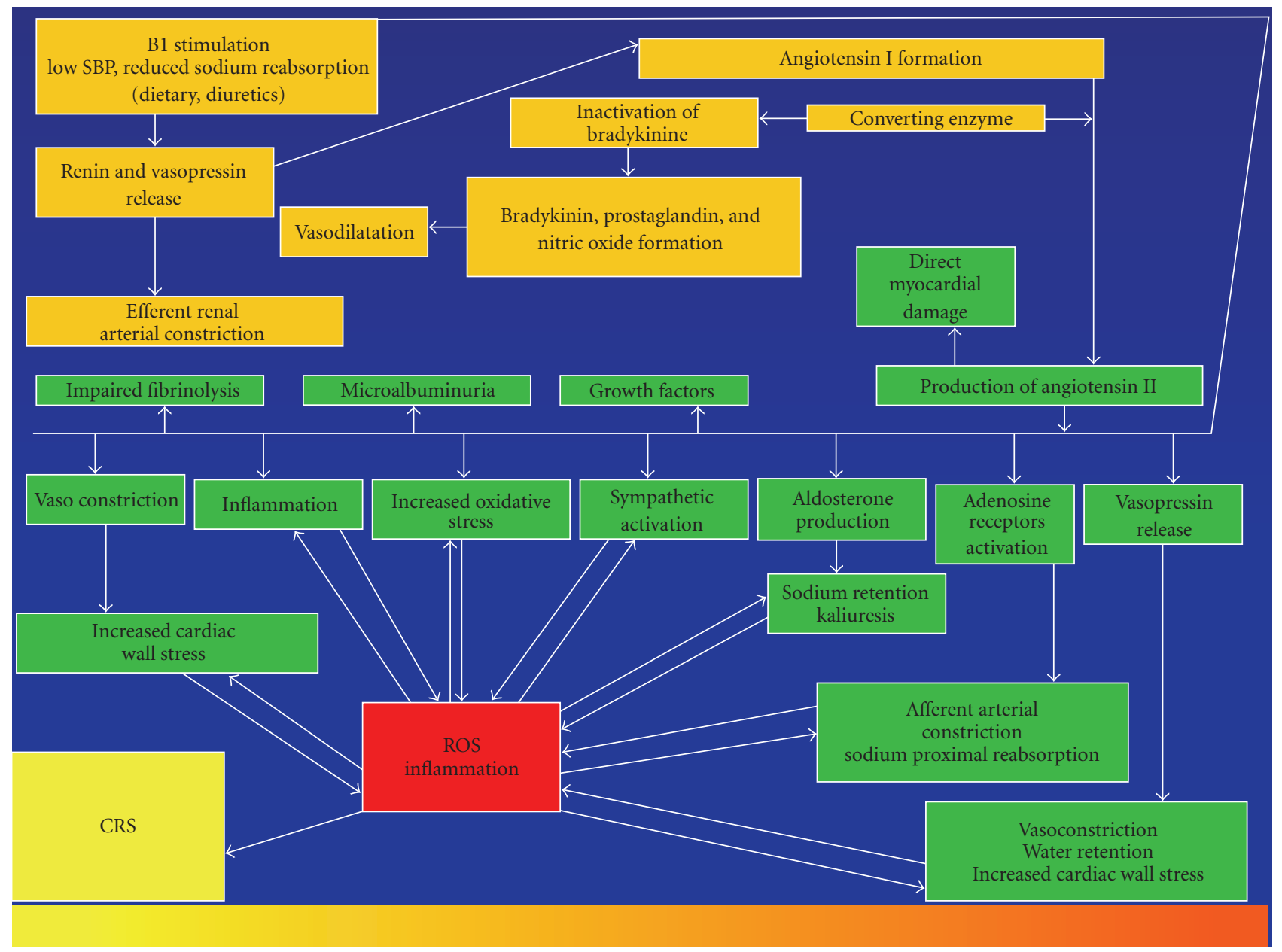

FIGURE 1: Pathophysiology of the cardiorenal syndrome. SBP: Systolic Blood Pressure; ROS: Reactive Oxygen Species; CRS: Cardiorenal Syndrome.

receptor antagonists, and ultrafiltration). As a result of the above, treatment of CRS patients is still quite empirical.

Generally, managing the patient with acute CRS often involves making therapeutic choices that are mutually contradictory. Because one is attempting to treat volume overload and congestion, the aggressive use of diuretics and volume depletion directly impairs renal function. Inhibitors of the RAAS (Angiotensin-converting enzyme inhibitors or angiotensin receptor blockers), although they are cardiorenal protective, can lead to temporary worsening of renal function. On the other hand, in order to preserve renal function, it is preferable to replace intravascular volume and provide a salt load, but these measures directly deteriorate cardiac congestion. Not surprisingly, many patients are discharged from hospital either still volume loaded or markedly worse in terms of renal function. That leads to a high readmission rate for patients recently discharged from hospital with heart or renal failure [2].

The management of the patient with ADHF and symptomatic congestion usually focuses on symptomatic relief and rapid removal of fluid. However, no therapies focused mainly on symptomatic relief or fluid removal have demonstrated any benefit on improving survival or attenuating the progression of the disease. This emphasizes the importance of instituting or optimizing disease-modifying therapy as soon as possible. These include, wherever they are appropriate, optimal doses of beta-blockers, angiotensin modulators, and aldosterone antagonists. All of these therapies, when used cautiously, will help to improve survival and reduce hospitalization rate. However, their effect on renal function and the hemodynamic status during acute decompensation will need close monitoring [2]. Table 1 summarizes some practical recommendations for the management of ADHF patients with type 1 CRS.

Body weight of the patient is the single most important indicator while managing the CRS. The patient needs continuous hemodynamic monitoring, especially if he has low blood pressure and uncertain filling pressure. Moderate restriction of daily salt intake $\leq 2 \mathrm{gr}$ is recommended. It is also better to restrict the dietary fluid intake to $1000 \mathrm{ml}-$ $1500 \mathrm{ml}$ or less than $1000 \mathrm{ml}$ per 24 hours if the patient is hyponatremic. A few cases with low filling pressure and low 
TABle 1: Managing cardiorenal syndrome: Practical recommendations.

(1) Restrict fluid and sodium intake

(2) Increase furosemide dose

(3) Use continuous intravenous furosemide

(4) Add thiazides or metolazone

(5) Add renoprotective dopamine at $2-3 \mathrm{mcg} / \mathrm{kg} / \mathrm{min}$

(6) Add inotrope or vasodilator (according to systolic blood pressure)

(7) Start ultrafiltration

(8) Insert intra-aortic balloon pump

(9) Insert another device

blood pressure may need volume expansion [16]. Drugs that impair kidney function should be avoided (e.g., NSAIDs), or their dosage should be adjusted according to the existing GFR (e.g., antibiotics).

3.1. Diuretics. Diuretic agents have long been considered to be an initial and essential part of the management of the CRS patients. However, limited clinical trial data suggest their beneficial role. The importance of diuretic agents is illustrated by data from the ADHFNR (Acute Decompensated Heart Failure National Registry), which revealed that $80.8 \%$ of patients enrolled in the registry were on chronic diuretic therapy at the time of admission while $88 \%$ were treated acutely with an intravenous diuretic during their admission for ADHF [9].

Loop, thiazide, and potassium-sparing diuretic agents cause diuresis and natriuresis in about 20 minutes after administration, and therefore they provide effective shortterm symptomatic relief. Nevertheless, they are not free from drawbacks, causing long-term detrimental cardiovascular effects. More specifically, they lead to activation of the neurohormonal system, indirectly deteriorate the function of the left ventricle, and increase systemic vascular resistance, plasma renin, aldosterone activity and plasma levels of neurohormones such as norepinephrine and arginine vasopressin. Through the above mechanisms, they result in promoting renal dysfunction, thus increasing the risk of mortality $[17,18]$.

In the absence of definitive data, patients with volume overload and nonhypotension should receive loop diuretics (slow high intravenous doses to minimize ototoxicity) or thiazides to alleviate symptoms. Despite the judicious use of loop diuretics, we should be very careful, because in the setting of ADHF and polypharmacy, such as the concomitant use of vasodilators, diuretics can cause hypotension in patients with systolic dysfunction and decreased cardiac preload.

A major problem the physicians have to face while treating patients with CRS is diuretic resistance, which is an indicator of poor prognosis in patients with CHF. It is described as a clinical state in which the diuretic response is diminished or lost before the therapeutic goal of relief from congestion has been reached. Many factors may be responsible for diuretic resistance, such as delayed intestinal absorption of oral drugs due to mucosal edema, decreased renal perfusion, decreased diuretic excretion into the urine, inadequate drug dosing, the concomitant use of NSAIDs, which inhibit the synthesis of vasodilator and natriuretic prostaglandins, and finally dietary noncompliance (e.g., excess salt intake) $[3,16,19]$.

Diuretic resistance is a common entity in the managing of patients with CRS. The braking phenomenon or shortterm tolerance means that the response to the diuretic is diminished after the first dose has been administered. This effect is treated by a continuous infusion of furosemide, rather than bolus doses, starting at $5 \mathrm{mg} / \mathrm{dl}$ to $10 \mathrm{mg} / \mathrm{dl}$, following an intravenous thiazide diuretic (the combination of loop diuretic and thiazide diuretic can cause sequential nephron blockade of sodium reabsorption). However, combination therapy requires careful monitoring, as it may lead to excessive sodium and potassium loss [20]. The continuous intravenous infusion in contrast to bolus injections of loop diuretics in diuretic-refractory patients seems to maintain a more optimal and effective rate of drug delivery to the renal tubules and in turn inhibits sodium reabsorption more consistently. A Cochrane review examined eight trials comparing continuous infusion of a loop diuretic with bolus injections in 254 patients with CHF. The urine output was significantly greater in patients given continuous infusion, the incidence of ototoxicity was less, and the duration of hospitalization was significantly shortened [21].

Several factors should be taken into account when deciding the diuretic dose in patients with refractory edema. It is important to remember that diuretics do not have a smooth dose-response curve: no natriuresis occurs until a threshold rate of drug excretion is achieved. Therefore, a patient who does not respond to $20 \mathrm{mg}$ of furosemide may not be exceeding this threshold, and the dose should be doubled rather than giving the same dose twice a day. In addition, the patient should cut down on his daily sodium intake, because high sodium can prevent net fluid loss even though adequate diuresis is being achieved. We should also consider the need for initial intravenous diuretic therapy in order to avoid the poor oral availability (only about $50 \%$ or less of oral furosemide is absorbed in edematous states) [3]. Table 2 summarizes some recommendations about the use of loop diuretics in heart failure patients according to the renal function [22].

It is also very important to mention that aggressive diuretic therapy at this stage could promote diuretic induced hypovolemia, exaggerating any pre-existing renal insufficiency as seen in acute CRS. Therefore a progressive and gradual diuresis as opposed to an aggressive and immediate one is recommended, especially in type 1 CRS [23].

Another approach to induce the efficacy of intravenous furosemide is to add salt-poor albumin in patients with low serum albumin levels. The furosemide-albumin complex is believed to deliver more diuretic to the kidney, primarily by staying in the vascular space. Studies have shown that adding salt-poor albumin substantially increased sodium excretion [24]. Finally, optimizing diuresis with the simultaneous use of hypertonic saline and diuretics has been studied and found 
TABle 2: Pharmacokinetics of loop diuretics according to the renal function in heart failure patients. IV: intravenous; CrCl: Creatinine Clearance.

\begin{tabular}{|c|c|c|c|c|c|c|c|}
\hline \multirow[b]{3}{*}{ Diuretic } & $\begin{array}{l}\text { Moderate } \\
\text { renal } \\
\text { insufficiency }\end{array}$ & \multicolumn{2}{|c|}{$\begin{array}{l}\text { Severe renal } \\
\text { insufficiency }\end{array}$} & \multicolumn{3}{|c|}{$\begin{array}{l}\text { Heart } \\
\text { failure }\end{array}$} & \\
\hline & \multicolumn{3}{|c|}{ Maximal intravenous dose (mg) } & \multirow[t]{2}{*}{$\begin{array}{l}\text { IV Loading dose } \\
(\mathrm{mg})\end{array}$} & \multicolumn{3}{|c|}{ Infusion rate $(\mathrm{mg} / \mathrm{hr})$} \\
\hline & & & & & $\begin{array}{c}\mathrm{CrCl} \\
<25 \mathrm{ml} / \mathrm{min} \\
\end{array}$ & $\begin{array}{c}\mathrm{CrCl} \\
25-75 \mathrm{ml} / \mathrm{min} \\
\end{array}$ & $\begin{array}{c}\mathrm{CrCl} \\
>75 \mathrm{ml} / \mathrm{min} \\
\end{array}$ \\
\hline Furosemide & $80-160$ & $160-200$ & $40-80$ & 40 & 20 then 40 & 10 then 20 & 10 \\
\hline Bumetanide & $4-8$ & $8-10$ & $1-2$ & 1 & 1 then 2 & 0.5 then 1 & 0.5 \\
\hline Torsemide & $20-50$ & $50-100$ & $10-20$ & 20 & 10 then 20 & 5 then 10 & 5 \\
\hline
\end{tabular}

successful at relieving signs and symptoms of congestion [25].

\subsection{ACE Inhibitors and Angiotensin Receptor}

Blockers (ARBs). Inhibitors of the RAAS are the key component in the management of patients with left ventricular systolic dysfunction. They improve survival in patients with heart failure and also prevent progressive renal insufficiency in diabetic nephropathy and other forms of chronic kidney disease. Nevertheless, in acute CRS these drugs should be used cautiously in patients with an underlying renal disease, because they may be associated with elevations in serum creatinine levels [26]. Although physicians frequently avoid or discontinue these drugs for fear of deteriorating renal function, the rise in serum creatinine levels after the initiation of an ACE inhibitor may identify a subgroup of patients who will achieve the greatest benefit from their use. Discontinuation of the ACEs because of renal dysfunction identified a patient group with heart failure who had an increased mortality risk [27].

Most trials that confirmed the benefits from the administration of ACE inhibitors, such as SOLVD [28], excluded patients with serum creatinine concentrations greater than $2 \mathrm{mg} / \mathrm{dl}$. The Cooperative North Scandinavian Enalapril Survival Study (CONSENSUS), in patients with severe heart failure, included patients with renal dysfunction, but only if their serum creatinine concentrations were no higher than $3.4 \mathrm{mg} / \mathrm{dl}$. The subgroup of patients with creatinine levels higher than $2 \mathrm{mg} / \mathrm{dl}$ showed evidence of improved outcomes when treated with an ACE inhibitor. CONSENSUS also demonstrated that patients with the most severe heart failure had a substantial increase in creatinine levels $(>30 \%)$, when an ACE inhibitor was added to their treatment, independent of their baseline renal function, while few patients needed to stop therapy [29]. To reduce the incidence of renal deterioration, patients should be started on the lowest dose of an ACE inhibitor, when the patient is considered not to be dehydrated, and concomitant use of NSAIDs should be avoided [15]. In addition, dosage up titration should be done very carefully. ACE inhibitor therapy in patients with baseline renal dysfunction is associated with significant long-term benefits and should be used in clinical routine, unless they are contraindicated (e.g., bilateral or high-grade renal artery stenosis and/or ACE/ARBs-induced hypotension) [30]. An effective approach is to continue these agents during hospitalization for DHF, despite an increase in creatinine levels, as long as renal dysfunction is not steadily impaired and severe hyperkalemia does not develop. ACE inhibitors are not usually related to worsening renal function in these patients. However, an expert physician should evaluate extreme clinical situations, such as cardiogenic shock or acute renal failure.

3.3. Low-Dose Dopamine. In clinical practice, low renalprotective doses of dopamine are commonly used in combination with diuretic therapy, although available data do not clearly support favorable effects on renal function. It is supposed that dopamine increases kidney blood flow, blunts the effects of norepinephrine and aldosterone, and, when given in low doses, promotes renal vasodilatation [31]. A prospective, double-blind, randomized, controlled study to investigate the effect of low-dose dopamine concluded that it can worsen renal perfusion in patients with acute renal failure, which adds to the trend to abandon the routine use of low-dose dopamine in critically ill patients [32].

A clinical trial (DAD-HF) by Triposkiadis et al. was announced in the Heart Failure Society of America Meeting 2009. It compared the effects of dopamine plus low-dose furosemide versus high-dose furosemide alone on kidney function and subjective perception of dyspnoea in ADHF. There were no differences in urine output or in dyspnoea score, but those patients who were treated with dopamine plus low-dose furosemide were less likely to have their renal function worsened at 24 hours or to develop hypokalemia. Although dopamine has been forgotten for a long time, it now seems that it may be undergoing a revival [33].

3.4. Inotropes. If the deteriorating renal function is thought to be related primarily to low cardiac output and subsequent reduced renal perfusion, positive inotropic agents (dobutamine, phosphodiesterase inhibitors, and levosimendan) may be used. These agents should be given only for low cardiac output states, for a short term and under close monitoring, as they may increase the risk of arrythmias. In both acute and chronic heart failure, inotropic drugs compared with placebo and vasodilators, have been related to an increased risk of mortality and other adverse cardiac events. Until more data are available, inotropic therapy 
should be reserved for patients with clinical evidence of severe low cardiac output (candidates for bridging to more definite therapy), in which vasodilatory agents cannot be administrated due to low systemic pressure or low systemic vascular resistance [34, 35].

Levosimendan belongs to a promising new class of inotropic agents called "calcium sensitizers." A randomized trial showed a moderate or marked improvement in the patient's overall assessment of patients treated with levosimendan [36]. An experimental study by Zager et al. showed that levosimendan protects against ischemic acute renal failure due to severe renal vasoconstriction, in critical situations such as sepsis or acute heart failure [37].

3.5. Vasodilators and Natriuretic Peptide. Vasodilators such as intravenous nitroglycerin or nesiritide (recombinant human B-type atrial natriuretic peptide) have been shown to be much less deleterious to kidney function, especially when used at low doses that do not reduce blood pressure. Vasodilators can rapidly decrease ventricular filling pressures and central venous pressures and reduce myocardial oxygen consumption. Intravenous nitroglycerine is a vasodilator used to relieve pulmonary congestion in patients with ADHF. Frequent dose titration of intravenous nitroglycerine according to systemic blood pressure is necessary in order to achieve the desired hemodynamic effects and symptomatic relief. The decrease in venous pressure may be beneficial in reducing transrenal perfusion pressure. But still it is not clear whether intravenous nitroglycerine has long-term benefits on kidney function or survival [2].

B-type natriuretic peptide (BNP) is synthesized in the ventricular myocardium in response to overload and wall stress. BNP dilates arteries and veins, induces sodium excretion, and suppresses the RAAS. Nesiritide, a synthetic BNP, is an effective vasodilator with a mild diuretic action. Its administration results in venous, arterial, and coronary vasodilatation, decreasing the cardiac preload and afterload, which in turn increases cardiac output without direct inotropic effects. These hemodynamic effects are accompanied by natriuresis and diuresis, although the latter responses may be quantitatively smaller than those in normal subjects and seem to be blunted in patients with more severe heart failure. Nevertheless, creatinine clearance was not improved by nesiritide, even in patients who showed satisfactory natriuresis and diuresis $[38,39]$.

In the setting of CRS, renal effects of nesiritide were first described by Wang and colleagues. They designed and implemented a crossover clinical trial in which 15 participants received a 24-hour infusion of nesiritide according to the recommended bolus and infusion regimen and a 24hour infusion of placebo on consecutive days, but in random order. They showed that nesiritide did not affect GFR, renal plasma flow, urine output, or sodium excretion [40]. The Vasodilatation in the Management of Acute Congestive Heart Failure (VMAC) trial assessed the impact of early nesiritide infusion on symptoms and pulmonary congestion in patients with DHF. A total of 489 patients with renal insufficiency received either nesiritide or nitroglycerin. At 24 hours, $83 \%$ of the patients with renal insufficiency and $91 \%$ of patients without renal insufficiency who were treated with nesiritide reported improvements in dyspnoea. Nesiritide might promote symptom improvement in heart failure patients with renal dysfunction but has no effect on kidney function [41]. A substudy of the Follow-Up Serial Infusions of Nesiritide trial (FUSION I) demonstrated that in heart failure patients who were at high risk for CRS, infusion of nesiritide at two doses $(0.005 \mu \mathrm{g} / \mathrm{kg} / \mathrm{ml}$ or $0.01 \mu \mathrm{g} / \mathrm{kg} / \mathrm{ml})$ was well tolerated with no deterioration of kidney function [42]. The serial infusion of nesiritide (FUSION II) trial was a study designed to look at intermittent infusion of nesiritide in patients with severe heart failure. Infusions were given either once or twice weekly over 12 weeks. This study demonstrated no significant effect on outcome or quality of life, but there was an effect on the kidney: an increasing serum creatinine level of more than $0.5 \mathrm{mg} / \mathrm{dl}$ was favorably affected by nesiritide [43].

Although first data show that low doses of nesiritide are potentially renal protective in the difficult clinical situation of treating patients with ADHF at risk for CRS, additional outcome information on the efficacy and safety of nesiritide is needed before it becomes an established therapy.

3.6. Ultrafiltration. The use of ultrafiltration is another potential therapeutic procedure in patients with diuretic resistance, which can alleviate volume overload. Ultrafiltration is a convective method for removing fluid and smallmolecular-weight compounds from the circulatory system across a semipermeable membrane in response to a transmembrane pressure gradient. Conventional ultrafiltration requiring central venous access is more frequently used, especially if the patient is extremely edematous [44]. The hemodynamic changes produced by ultrafiltration are modest. The reduction in water is accompanied by decreases in right atrial pressure and wedge pressure. Cardiac output and stroke volume do not change or rise slightly [45]. Compared to loop diuretics, ultrafiltration is more efficient in removing sodium, while the neurohormonal activation is less for the same degree of volume reduction. An important point is that weight loss is sustained relatively to furosemide treatment. The typical volume of water removed per ultrafiltration session is 3 to 4 lt. Loop diuretics should be discontinued for the days the patient is receiving ultrafiltration in order to minimize electrocyte abnormalities and neurohormonal activation [46].

Several trials have evaluated the use and efficacy of ultrafiltration. Seven centers participated in a pilot randomized controlled study, RAPID CHF (Randomized Controlled Trial of Ultrafiltration for Decompensated Congestive Heart Failure: the Relief for Acutely Fluid-Overloaded Patients with Decompensated Congestive Heart Failure), which compared a single 8-hour ultrafiltration intervention to usual care of 40 patients hospitalized with DHF. Total fluid removal at 24 hours was greater with ultrafiltration than with the usual care, with a trend towards greater weight loss at 24 hours in the ultrafiltration group [47].

Ultrafiltration can potentially manage worsening renal function and decreased urine output despite escalating 
doses of diuretics or diuretic resistance in severe heart failure. In the Ultrafiltration versus Intravenous Diuretics for patients Hospitalized for Acute Decompensated Heart Failure (UNLOAD) trial, patients with ADHF were randomly assigned to ultrafiltration with flows of up to $500 \mathrm{ml} / \mathrm{h}$ versus standard intravenous diuretics. The ultrafiltration group showed a greater weight loss and greater fluid removal at 48 hours, although the changes in dyspnoea score did not differ and both groups improved. The rates of re-hospitalization and the total days of hospitalization were significantly lower in the ultrafiltration group at a 3-month follow-up. However, preliminary data suggested that there was not a significant protective effect of ultrafiltration on kidney function. Surprisingly, there was no relationship between the amounts of fluid removal versus changes in serum creatinine levels, suggesting that other factors not associated with volume are responsible for the deterioration in renal function in CRS [48].

Overall, compared with the use of intravenous diuretics with or without combined vasoactive therapy, ultrafiltration provides a quick and predictable removal of fluid that is free of induced electrocytes abnormalities and associated consequences. On the other hand, ultrafiltration may be related to high daily cost as well as the need for large vein access and greater patient supervision [44].

3.7. Vasopressin Antagonists. Arginine vasopressin (AVP) or antidiuretic hormone is secreted by the posterior pituitary gland in response to hyperosmolality or volume depletion. Its actions are mediated by three types of receptors: $V_{1 \mathrm{~A}}$, $V_{1 B}$, and $V_{2} . V_{2}$ receptors are located in the distal tubules of the kidney and the collecting duct, and they provoke vasoconstriction and water reabsorption through aquaporin channels in the tubules. In heart failure, secretion of AVP may be enhanced due to low blood pressure or diminished arterial volume. Excess AVP can lead to hyponatremia. Selective $V_{2}$ antagonists (vaptans), such as tolvaptan and conivaptan, can effectively mobilize free water clearance and aquaresis and increase the serum sodium in those that are hyponatremic [48].

Some studies have reported a powerful aquaretic effect without renal impairment in patients with ADHF treated with tolvaptan. In the Acute and Chronic Therapeutic Impact of a Vasopressin Antagonist (ACTIV) trial, patients with acute heart failure showed a greater decrease in body weight, an increase in urine output and a slight increase in serum sodium at 24 hours receiving tolvaptan compared to those receiving placebo or standard therapy [49]. The much larger Efficacy of Vasopressin Antagonist in Heart Failure Outcome Study with Tolvaptan (EVEREST) confirmed the efficacy of early administration of vasopressin antagonists in decreasing mean body weight and improving dyspnoea. It comprised 4133 patients who were hospitalized for acute heart failure and then they were followed up during longterm treatment. Long-term outcomes of the patients did not differ between vasopressin antagonist and the placebo groups. This suggests that vaptans when used in the context of acute heart failure can modify kidney response to water retention. But still it does not favorably influence remodeling of heart and kidneys over the long term towards recovery $[50,51]$.

3.8. Adenosine Antagonists. Adenosine is generated by the breakdown of ATP and ADP in the renal tubules during the energy-requiring process of sodium excretion. As sodium excretion increases in conditions such as during diuretic therapy in sodium overload states (e.g., heart failure), extracellular adenosine concentrations rise and serve as a counterregulator trying to restore the balance between energy supply and demand. The elevated plasma adenosine levels observed in patients with heart failure can contribute to diuretic resistance and renal dysfunction. In the context of impaired tubular glomerular filtration, adenosine is released and binds to $A_{1}$ receptors to cause constriction of the afferent arterioles. This reduces renal blood flow and induces sodium reabsorption by the proximal tubules. $A_{1}$ adenosine receptor antagonists are novel agents that activate adenosine $A_{1}$ receptors and improve renal blood flow, promote diuresis, and increase sodium excretion.

The efficacy of an adenosine $A_{1}$ receptor antagonist in the treatment of patients with heart failure is still unsettled. Initial clinical studies seem to be quite controversial. Gottlieb et al. showed that the addition of BG9719 $\left(A_{1}\right.$ adenoside antagonist) to furosemide in patients with heart failure and volume overload significantly increased diuresis and prevented a decline in kidney function [14]. On the other hand, the results of PROTECT (A Placebo-controlled Randomized Study of the Selective $A_{1}$ Adenosine Receptor Antagonist Rolofylline for Patients Hospitalized With Acute Decompensated Heart Failure and Volume Overload to Assess Treatment Effect on Congestion and Renal Function) clinical trial that were recently announced in the ESC 2009 showed that rolofylline did not meet neither the primary efficacy endpoints (dyspnoea improvement, treatment failure) nor the secondary efficacy end points (death, cardiovascular or renal rehospitalization, or persistent renal impairment), while the overall safety profiles of the placebo and rolofylline groups were similar (rolofylline was associated with higher incidence of seizure and a trend towards a higher incidence of stroke) [52]. In addition, the REACH UP trial, a multicenter, international, randomized, double-blind, placebo-controlled study did not demonstrate any clear benefit of rolofylline on clinical status or renal function in patients with ADHF and recent or acute worsening renal function. Although there were fewer deaths or rehospitalizations at 60 days in the rolofylline-treated patients, the numbers were small and did not reach statistical significance [53]. Thus, larger clinical trials are required in order to determine their net effect on renal function and potential cardiovascular outcomes.

3.9. Targeted Renal Delivery of Drugs. Targeted renal delivery of drugs has been proposed to increase local drug concentration in the hopes of inducing renal effects or providing a previously unattained effect. Direct intrarenal delivery (to both renal arteries simultaneously) will eliminate renal first pass, resulting in less systemic exposure and reduction of serious side effects. Intrarenal delivery of fenoldopam (dopamine $\mathrm{D}_{1}$ agonist) was associated with a lower incidence 
of hypotension than intravenous fenoldopam. This is also true for intrarenal versus intravenous administration of nesiritide because BNP has high first-pass renal metabolism [54].

3.10. Anemia and Erythropoietin. Although the prevalence of anemia in the heart failure population has been subject to very wide variations, it is a long recognized, common, and well-studied complication of chronic kidney disease (CKD). Furthermore, cardiorenal anemia syndrome refers to the simultaneous presence of anemia, heart failure, and CKD that forms a pathologic triad with an adverse impact on morbidity and mortality [55]. Several studies have shown that when anemia is corrected with subcutaneous erythropoietin, the cardiac function improves, as assessed by measurement of the ventricular ejection fraction and oxygen utilization during maximal exercise [56]. However, anemia should not be aggressively corrected in the renal failure population [57]. Recently, we have shown that darbepoetin alpha attenuates deleterious effects of oxidative and nitrosative stress into the cardiovascular system of anemic patients with CHF, counteracts neurohormonal activation, and also improves cardiac function and exercise capacity $[58,59]$. The results of the Reduction of Events with Darbepoetin alpha in Heart Failure Trial (RED-HF trial) will determine the role and efficacy of treatment of anemia with darbepoetin alpha on the mortality and morbidity in heart failure subjects with symptomatic left ventricular systolic dysfunction and anemia [60].

\subsection{Cardiac Transplantation/Cardiac Assist}

Devices. Patients with CRS are rarely candidates for advanced heart failure therapy, such as cardiac transplantation or implantation of a left ventricular assist device, because of their high surgical risk and poor prognosis. Still, there are devices such as the intraaortic balloon pump, which are used in low cardiac output states and contribute to the hemodynamic stabilization of the patients and therefore preserve renal function.

\section{Conclusion}

The challenges in the management of acute CRS will worsen before they get better due to our success in improving survival in heart failure patients. In addition, growing numbers of patients will survive to reach the true endstage of heart failure. The previous focus on symptomatic treatment with increasing doses of diuretics and vasodilators, which met resistance, is now fading. The new focus should be to recognize the cardiorenal syndrome, recognize it early and treat the whole patient for long term. The optimization of heart failure therapy also preserves kidney function. Novel therapeutic options may offer additional opportunities to improve volume regulation, while preserving cardiac and renal function. A close cooperation of cardiologists, nephrologists, and internists is required, as well as a deeper understanding of the pathophysiology of the CRS, in order to establish an effective means of therapy in the future.

\section{References}

[1] H. L. Hillege, D. Nitsch, M. A. Pfeffer et al., "Renal function as a predictor of outcome in a broad spectrum of patients with heart failure," Circulation, vol. 113, no. 5, pp. 671-678, 2006.

[2] P. P. Liu, "Cardiorenal syndrome in heart failure: a cardiologist's perspective," Canadian Journal of Cardiology, vol. 24, supplement, pp. 25B-29B, 2008.

[3] C. Geisberg and J. Butler, "Addressing the challenges of cardiorenal syndrome," Cleveland Clinic Journal of Medicine, vol. 73, no. 5, pp. 485-491, 2006.

[4] D. L. Dries, D. V. Exner, M. J. Domanski, B. Greenberg, and L. W. Stevenson, "The prognostic implications of renal insufficiency in asymptomatic and symptomatic patients with left ventricular systolic dysfunction," Journal of the American College of Cardiology, vol. 35, no. 3, pp. 681-689, 2000.

[5] G. C. Fonarow, K. F. Adams, and W. T. Abraham, "ADHERE Investigators. Risk stratification for in-hospital mortality in heart failure using classification and regression tree (CART) methodology: analysis of 33,046 patients in the ADHERE registry," Journal of Cardiac Failure, vol. 9, supplement, p. S79, 2003, abstract no. 290.

[6] S. S. Gottlieb, W. Abraham, J. Butler et al., "The prognostic importance of different definitions of worsening renal function in congestive heart failure," Journal of Cardiac Failure, vol. 8, no. 3, pp. 136-141, 2002.

[7] C. Ronco, "Cardiorenal and renocardiac syndromes: clinical disorders in search of a systematic definition," International Journal of Artificial Organs, vol. 31, no. 1, pp. 1-2, 2008.

[8] C. Ronco, A. A. House, and M. Haapio, "Cardiorenal syndrome: refining the definition of a complex symbiosis gone wrong," Intensive Care Medicine, vol. 34, no. 5, pp. 957-962, 2008.

[9] C. W. Yancy, M. Lopatin, L. W. Stevenson et al., "ADHERE Scientific Advisory Committee and Investigators. Clinical presentation, management and in- hospital outcomes of patients admitted with acute decompansated heart failure with preserved systolic function: a report from the Acute Decompansated Heart Failure National Registry (ADHERE) Database," Journal of the American College of Cardiology, vol. 47, pp. 76-84, 2006.

[10] C. Heymes, J. K. Bendall, P. Ratajczak et al., "Increased myocardial NADPH oxidase activity in human heart failure," Journal of the American College of Cardiology, vol. 41, no. 12, pp. 2164-2171, 2003.

[11] L. G. Bongartz, M. J. Cramer, P. A. Doevendans et al., "The severe cardiorenal syndrome: 'Guyton revisited," European Heart Journal, vol. 26, no. 1, pp. 11-17, 2005.

[12] G. W. Moe, J. L. Rouleau, Q. T. Nguyen, P. Cernacek, and D. J. Stewart, "Role of endothelins in congestive heart failure," Canadian Journal of Physiology and Pharmacology, vol. 81, no. 6, pp. 588-597, 2003.

[13] C. R. Lee, M. L. Watkins, J. H. Patterson et al., "Vasopressin: a new target for the treatment of heart failure," American Heart Journal, vol. 146, no. 1, pp. 9-18, 2003.

[14] S. S. Gottlieb, D. C. Brater, I. Thomas et al., "BG9719 (CVT124), an A1 adenosine receptor antagonist, protects against the decline in renal function observed with diuretic therapy," Circulation, vol. 105, no. 11, pp. 1348-1353, 2002.

[15] M. G. Shlipak, "Pharmacotherapy for heart failure in patients with renal insufficiency," Annals of Internal Medicine, vol. 138, no. 11, pp. 917-924, 2003.

[16] G. Francis, "Acute decompensated heart failure: the cardiorenal syndrome," Cleveland Clinic Journal of Medicine, vol. 73, supplement 2, pp. S8-S13, 2006. 
[17] M. R. Costanzo, J. T. Heywood, T. DeMarco et al., "Impact of renal insufficiency and chronic diuretic therapy on outcome and resource utilization in patients with acute decompensated heart failure," Journal of the American College of Cardiology, vol. 43, supplement 1, p. A180, 2004.

[18] J. Bayliss, M. Norell, R. Canepa-Anson, G. Sutton, and P. PoleWilson, "Untreated heart failure: clinical and neuroendocrine effects of introducing diuretics," British Heart Journal, vol. 57, no. 1, pp. 17-22, 1987.

[19] B. K. Krämer, F. Schweda, and G. A. J. Riegger, "Diuretic treatment and diuretic resistance in heart failure," American Journal of Medicine, vol. 106, no. 1, pp. 90-96, 1999.

[20] J. R. Oster, M. Epstein, and S. Smoller, "Combined therapy with thiazide-type and loop diuretic agents for resistant sodium retention," Annals of Internal Medicine, vol. 99, no. 3, pp. 405-406, 1983.

[21] D. R. Salvador, N. R. Rey, G. C. Ramos, and F. E. Punzalan, "Continuous infusion versus bolus injection of loop diuretics in congestive heart failure," Cochrane Database of Systematic Reviews, no. 1, Article ID CD003178, 2004.

[22] D. C. Brater, "Diuretic therapy," The New England Journal of Medicine, vol. 339, no. 6, pp. 387-395, 1998.

[23] C. Ronco, M. Haapio, A. A. House, N. Anavekar, and R. Bellomo, "Cardiorenal Syndrome," Journal of the American College of Cardiology, vol. 52, no. 19, pp. 1527-1539, 2008.

[24] D. Fliser, I. Zurbrüggen, E. Mutschler et al., "Coadministration of albumin and furosemide in patients with the nephrotic syndrome," Kidney International, vol. 55, no. 2, pp. 629-634, 1999.

[25] M. H. Drazner and B. F. Palmer, "Hypertonic saline: a novel therapy for advanced heart failure?" American Heart Journal, vol. 145, no. 3, pp. 377-379, 2003.

[26] G. L. Bakris and M. R. Weir, "Angiotensin-converting enzyme inhibitor-associated elevations in serum creatinine: is this a cause for concern?" Archives of Internal Medicine, vol. 160, no. 5, pp. 685-693, 2000.

[27] M. Kittleson, S. Hurwitz, M. R. Shah et al., "Development of circulatory-renal limitations to angiotensin-converting enzyme inhibitors identifies patients with severe heart failure and early mortality," Journal of the American College of Cardiology, vol. 41, no. 11, pp. 2029-2035, 2003.

[28] S. Yusuf, "Effect of enalapril on survival in patients with reduced left ventricular ejection fractions and congestive heart failure," The New England Journal of Medicine, vol. 325, no. 5, pp. 293-302, 1991.

[29] S. Ljungman, J. Kjekshus, and K. Swedberg, "Renal function in severe congestive heart failure during treatment with Enalapril (the Cooperative North Scandinavian Enalapril Survival Study [CONSENSUS] trial)," American Journal of Cardiology, vol. 70, no. 4, pp. 479-487, 1992.

[30] J. Butler, D. E. Forman, W. T. Abraham et al., "Relationship between heart failure treatment and development of worsening renal function among hospitalized patients," American Heart Journal, vol. 147, no. 2, pp. 331-338, 2004.

[31] P. Marik, "Low-dose dopamine: a systematic review," Intensive Care Medicine, vol. 28, no. 7, pp. 877-883, 2002.

[32] A. Lauschke, U. Teichgräber, U. Frei, and K.-U. Eckardt, “ Low-dose' dopamine worsens renal perfusion in patients with acute renal failure," Kidney International, vol. 69, no. 9, pp. 1669-1674, 2006.

[33] M. Lainscak, A. P. Coletta, N. Sherwi, and J. G. F. Cleland, "Clinical trials update from the Heart Failure Society of America Meeting 2009: FAST, IMPROVE-HF, COACH galectin3 substudy, HF-ACTION nuclear substudy, DAD-HF, and
MARVEL-1," European Journal of Heart Failure, vol. 12, no. 2, pp. 193-196, 2010.

[34] U. Elkayam, G. Tasissa, C. Binanay et al., "Use and impact of inotropes and vasodilator therapy in hospitalized patients with severe heart failure," American Heart Journal, vol. 153, no. 1, pp. 98-104, 2007.

[35] G. M. Felker and C. M. O'Connor, "Inotropic therapy for heart failure: an evidence-based approach," American Heart Journal, vol. 142, no. 3, pp. 393-401, 2001.

[36] L. Lehtonen, "Levosimendan: a calcium-sensitizing agent for the treatment of patients with decompensated heart failure," Current Heart Failure Reports, vol. 1, no. 3, pp. 136-144, 2004.

[37] R. A. Zager, A. C. Johnson, S. Lund, S. Y. Hanson, and C. K. Abrass, "Levosimendan protects against experimental endotoxemic acute renal failure," American Journal of Physiology, vol. 290, no. 6, pp. F1453-F1462, 2006.

[38] L. S. Marcus, D. Hart, M. Packer et al., "Hemodynamic and renal excretory effects of human brain natriuretic peptide infusion in patients with congestive heart failure: a doubleblind, placebo-controlled, randomized crossover trial," Circulation, vol. 94, no. 12, pp. 3184-3189, 1996.

[39] W. T. Abraham, B. D. Lowes, D. A. Ferguson et al., "Systemic hemodynamic, neurohormonal, and renal effects of a steadystate infusion of human brain natriuretic peptide in patients with hemodynamically decompensated heart failure," Journal of Cardiac Failure, vol. 4, no. 1, pp. 37-44, 1998.

[40] D. J. Wang, T. C. Dowling, D. Meadows et al., "Nesiritide does not improve renal function in patients with chronic heart failure and worsening serum creatinine," Circulation, vol. 110, no. 12, pp. 1620-1625, 2004.

[41] J. B. Young, "Intravenous nesiritide vs nitroglycerin for treatment of decompensated congestive heart failure: a randomized controlled trial," Journal of the American Medical Association, vol. 287, no. 12, pp. 1531-1540, 2002.

[42] C. W. Yancy and A. Singh, "Potential applications of outpatient nesiritide infusions in patients with advanced heart failure and concomitant renal insufficiency (from the Follow-Up Serial Infusions of Nesiritide [FUSION I] Trial)," American Journal of Cardiology, vol. 98, no. 2, pp. 226-229, 2006.

[43] C. W. Yancy, H. Crum, B. M. Massie et al., "Safety and efficacy of out patient nesiritide in patients with advanced heart failure. Results of the serial infusion of nesiritide (FUSION II) trial," Circulation, vol. 1, pp. 9-16, 2008.

[44] B. E. Jaski and D. Miller, "Ultrafiltration in decompensated heart failure," Current Heart Failure Reports, vol. 2, no. 3, pp. 148-154, 2005.

[45] G. Marenzi, G. Lauri, M. Grazi, E. Assanelli, J. Campodonico, and P. Agostoni, "Circulatory response to fluid overload removal by extracorporeal ultrafiltration in refractory congestive heart failure," Journal of the American College of Cardiology, vol. 38, no. 4, pp. 963-968, 2001.

[46] B. A. Bart, A. Boyle, A. J. Bank et al., "Ultrafiltration versus usual care for hospitalized patients with heart failure: the relief for acutely fluid-overloaded patients with decompensated congestive heart failure (RAPID-CHF) trial," Journal of the American College of Cardiology, vol. 46, no. 11, pp. 2043-2046, 2005.

[47] M. R. Costanzo, M. E. Guglin, M. T. Saltzberg et al., "Ultrafiltration versus intravenous diuretics for patients hospitalized for acute decompensated heart failure," Journal of the American College of Cardiology, vol. 49, no. 6, pp. 675-683, 2007. 
[48] S. Nielsen, C.-L. Chou, D. Marples, E. I. Christensen, B. K. Kishore, and M. A. Knepper, "Vasopressin increases water permeability of kidney collecting duct by inducing translocation of aquaporin-CD water channels to plasma membrane," Proceedings of the National Academy of Sciences of the United States of America, vol. 92, no. 4, pp. 1013-1017, 1995.

[49] M. Gheorghiade, W. A. Gattis, C. M. O'Connor et al., "Effects of tolvaptan, a vasopressin antagonist, in patients hospitalized with worsening heart failure: a randomized controlled trial," Journal of the American Medical Association, vol. 291, no. 16, pp. 1963-1971, 2004.

[50] M. Gheorghiade, M. A. Konstam, J. C. Burnett Jr. et al., "Short-term clinical effects of tolvaptan, an oral vasopressin antagonist, in patients hospitalized for heart failure: the EVEREST clinical status trials," Journal of the American Medical Association, vol. 297, no. 12, pp. 1332-1343, 2007.

[51] M. A. Konstam, M. Gheorghiade, J. C. Burnett Jr. et al., "Effects of oral tolvaptan in patients hospitalized for worsening heart failure: the EVEREST outcome trial," Journal of the American Medical Association, vol. 297, no. 12, pp. 1319-1331, 2007.

[52] B. D. Weatherley, G. Cotter, H. C. Dittrich et al., "Design and rationale of the PROTECT study: a placebo-controlled randomized study of the selective A1 adenosine receptor antagonist rolofylline for patients hospitalized with acute decompensated heart failure and volume overload to assess treatment effect on congestion and renal function," Journal of Cardiac Failure, vol. 16, no. 1, pp. 25-35, 2010.

[53] S. S. Gottlieb, M. M. Givertz, M. Metra et al., "The effects of adenosine A1 receptor antagonism in patients with acute decompensated heart failure and worsening renal function: the REACH UP study," Journal of Cardiac Failure, vol. 16, no. 9, pp. 714-719, 2010.

[54] H. Madyoon, P. Terstein, D. Baim, L. Croushore, E. Anderson, and V. Mathur, "Different effects between intravenous and local renal delivery of fenoldopam on renal function and blood pressure: a randomized controlled trial," American Journal of Cardiology, vol. 94, supplement 6, pp. 22E-23E, 2004.

[55] A. Kazory and E. A. Ross, "Anemia: the point of convergence or divergence for kidney disease and heart failure?" Journal of the American College of Cardiology, vol. 53, no. 8, pp. 639-647, 2009.

[56] D. Silverberg, D. Wexler, M. Blum, Y. Wollman, and A. Iaina, "The cardio-renal anaemia syndrome: does it exist?" Nephrology Dialysis Transplantation, vol. 18, no. 8, supplement, pp. viii7-viii12, 2003.

[57] A. K. Singh, L. Szczech, K. L. Tang et al., "CHOIR Investigators. Correction of anemia with apoetin alpha in chronic kidney disease," The New England Journal of Medicine, vol. 355, pp. 2085-2098, 2006.

[58] J. T. Parissis, K. Kourea, F. Panou et al., "Effects of darbepoetin $\alpha$ on right and left ventricular systolic and diastolic function in anemic patients with chronic heart failure secondary to ischemic or idiopathic dilated cardiomyopathy," American Heart Journal, vol. 155, no. 4, pp. 751.e1-751.e7, 2008.

[59] J. T. Parissis, K. Kourea, I. Andreadou et al., "Effects of darbepoetin alpha on plasma mediators of oxidative and nitrosative stress in anemic patients with chronic heart failure secondary to ischemic or idiopathic dilated cardiomyopathy," American Journal of Cardiology, vol. 103, no. 8, pp. 1134-1138, 2009.

[60] J. J. McMurray, I. S. Anand, R. Diaz et al., "RED-HF Committees and Investigators. Design of the Reduction of Events with Darbepoetin alpha in Heart Failure (RED-HF): a phase III, anaemia correction, morbidity-mortality trial," European Journal of Heart Failure, vol. 11, no. 8, pp. 795-801, 2009. 


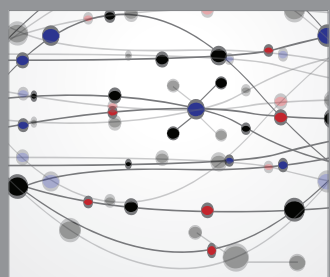

The Scientific World Journal
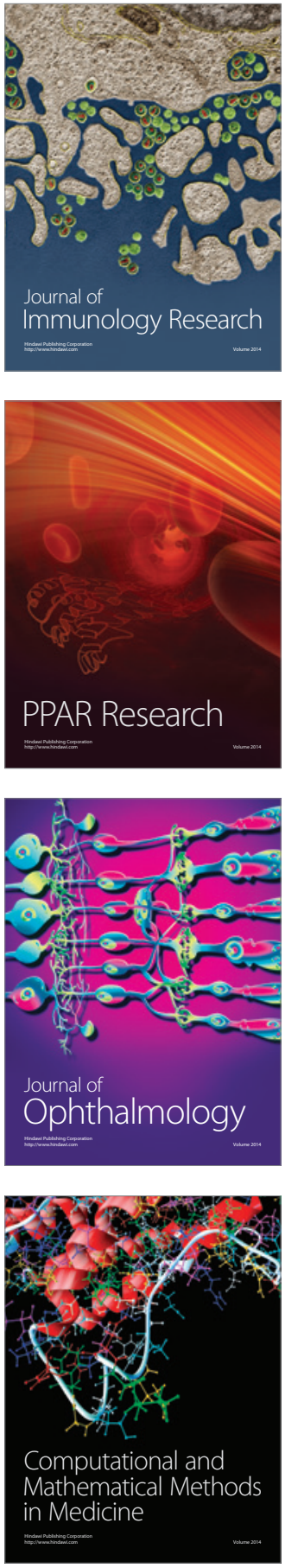

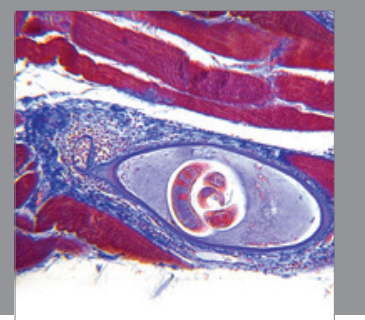

Gastroenterology

Research and Practice
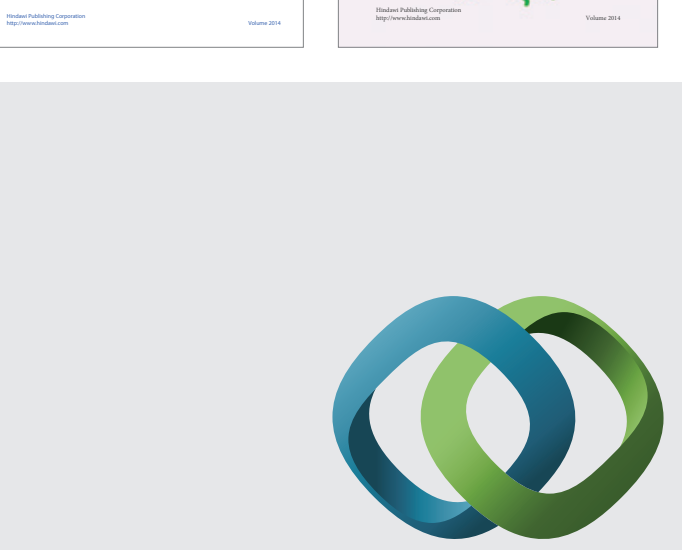

\section{Hindawi}

Submit your manuscripts at

http://www.hindawi.com
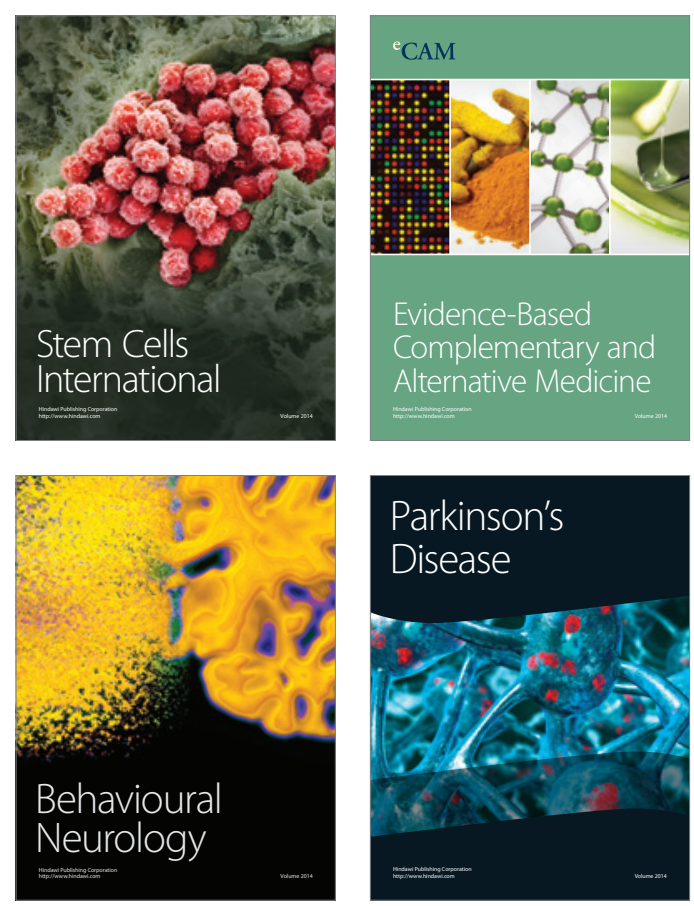

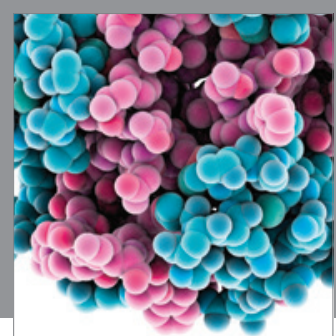

Journal of
Diabetes Research

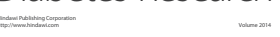

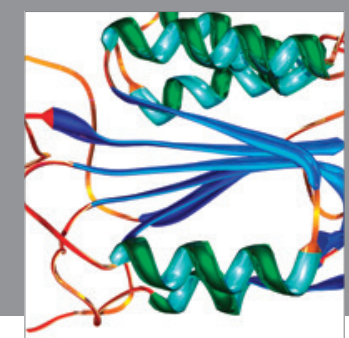

Disease Markers
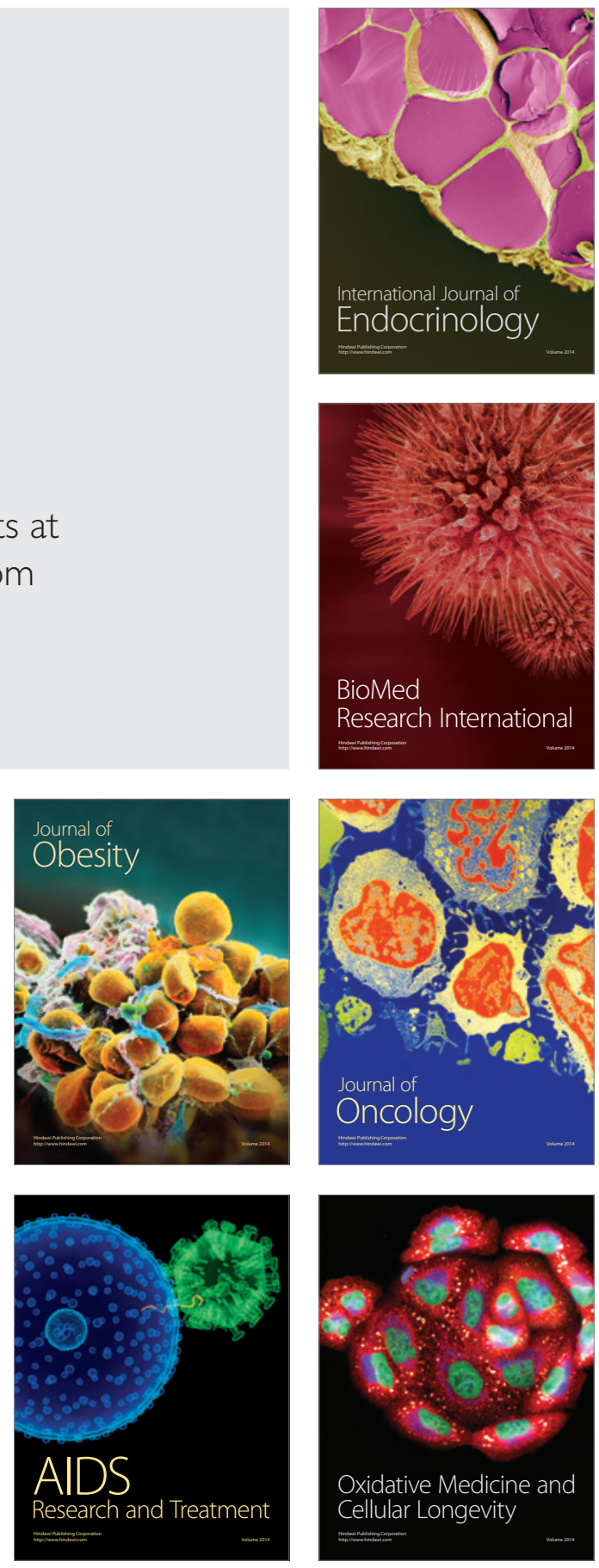